\title{
Generating Functions for k-Hypergeometric Functions
}

\author{
Duriye Korkmaz-Duzgun ${ }^{1 *}$, Esra Erkus-Duman ${ }^{2}$ \\ ${ }^{1}$ Kafkas University, Faculty of Economics and Administrative Sciences, Department of Business \\ Administration, Central Campus TR-36100 Kars, Turkey. \\ ${ }^{2}$ Gazi University, Faculty of Science, Department of Mathematics, 06500 Teknikokullar, Ankara, Turkey. \\ * Corresponding author. Tel.: +90474 2251150-1772; email: dkduzgun@kafkas.edu.tr, \\ dryekorkmaz@gmail.com \\ Manuscript submitted February 15, 2019; accepted April 12, 2019. \\ doi: 10.17706/ijapm.2019.9.3.119-126
}

\begin{abstract}
In mathematics, generating functions are important way to transform formal power series into functions and to analyze asymptotic properties of sequences. On the other hand, hypergeometric function is a special function represented by the hypergeometric series. Gauss, Confluent, Appell, Lauricella, and Horn functions are as an example of hypergeometric functions. In this article, we will introduce k-hypergeometric functions which are extensions of the Gauss hypergeometric functions including k- Pochhammer symbol. We first give an identity for k-Pochhammer symbol and certain linear generating functions for k-hypergeometric functions. Then we derive a family of multilinear and multilateral generating functions for these functions. In the main theorems, specially, bilateral generating function relations are obtained by applying extended multivariable hypergeometric functions and Cesáro Polynomials. Additionally we present bilinear generating functions for k-hypergeometric functions.
\end{abstract}

Key words: Hypergeometric functions, generating functions, multilinear and multilateral generating functions, k-hypergeometric functions, $k$-Pochhammer symbol.

\section{Introduction}

The hypergeometric functions are important for obtaining various properties, such as, integral representation, generating functions, solution of Gauss differential equations.

We aim at deriving some generating functions for a family of the $k$-hypergeometric functions defined by (see [1]):

$$
\begin{aligned}
& { }_{2} F_{1, k}=F_{k}(\alpha, \beta ; \gamma ; z):=\sum_{n=0}^{\infty} \frac{(\alpha)_{n, k}(\beta)_{n, k}}{(\gamma)_{n, k} n !} z^{n} \\
& \quad(\alpha, \beta, \gamma \in C ; \quad \gamma \neq 0,-1,-2, \ldots ;|z|<1 \text { and } k>0)
\end{aligned}
$$

in terms of the following Pochhammer $k$-symbol $(\lambda)_{v, k}(\lambda, v \in C)$ defined by [2]:

$$
\begin{aligned}
& (\lambda)_{v, k}:=\frac{\Gamma_{k}(\lambda+v k)}{\Gamma_{k}(\lambda)} \\
& =\left\{\begin{array}{cl}
1 & ; v=0, \lambda \in C-\{0\} \\
\lambda(\lambda+k)(\lambda+2 k) \ldots(\lambda+(n-1) k) & ; v=n, n \in N, k>0 \lambda \in C-k Z^{-}
\end{array}\right.
\end{aligned}
$$


where the $k$-gamma function $\Gamma_{k}(z)$ was introduced in [2] as follows:

$$
\Gamma_{k}(z):=\int_{0}^{\infty} t^{z-1} e^{\frac{-t^{k}}{k}} d t \quad \operatorname{Re}(z)>0
$$

Here, and in what follows, we respectively denote by $R$ and $C$ the sets of real and complex numbers and $N_{0}:=N \bigcup\{0\},(N=1,2,3, \ldots)$ and $Z:=Z^{-} \cup\{0\},\left(Z^{-}=-1,-2,-3, \ldots\right)$.

Clearly, in the special case $k=1$ equations (1), (2), and (3) are reduces to the usual hypergeometric function, Pochhammer symbol, and gamma function given by respectively [3]:

$$
\begin{gathered}
F(\alpha, \beta ; \gamma ; z):=\sum_{n=0}^{\infty} \frac{(\alpha)_{n}(\beta)_{n}}{(\gamma)_{n} n !} z^{n} ; \quad(|z|<1), \\
(\lambda)_{v}=\frac{\Gamma(\lambda+v)}{\Gamma(\lambda)}=\left\{\begin{array}{c}
1 \\
\lambda(\lambda+1)(\lambda+2) \ldots(\lambda+(n-1)) ; v=n, n \in N, \lambda \in C
\end{array},\right. \\
\Gamma(z)=\int_{0}^{\infty} t^{z-1} e^{-t} d t, \quad \operatorname{Re}(z)>0 .
\end{gathered}
$$

The main aim of this paper is to derive various classes of multilinear and multilateral generating functions for $k$-hypergeometric functions given by (1). We also give special cases of generating functions presented in this article.

\section{Generating Functions}

In this section, we derive a property of $k$-Pochhammer symbol and obtain generating function relations for $k$-hypergeometric functions.

Lemma 2.1. We have following identity for k-Pochhammer symbol given by (2):

$$
\frac{(-n)_{r, k}}{(-1)^{r}}=\frac{k^{r}\left(\frac{n}{k}\right) !}{\left(\frac{n}{k}-r\right) !}
$$

Proof. If we use $k$-Pochhammer symbol definition given by (2), then

$$
\begin{aligned}
(-n)_{r, k} & =(-n)(-n+k)(-n+2 k) \ldots(-n+k(r-1)) \\
& =\frac{(-1)^{r} k^{r}\left(\frac{n}{k}\right)\left(\frac{n}{k}-1\right) \ldots\left(\frac{n}{k}-(r-1)\right)\left(\frac{n}{k}-r\right) \ldots 321}{\left(\frac{n}{k}-r\right) \ldots 321} \\
& =\frac{(-1)^{r} k^{r}\left(\frac{n}{k}\right) !}{\left(\frac{n}{k}-r\right) !}
\end{aligned}
$$


which completes the proof.

Theorem 2.1. We have the following generating function for the k-hypergeometric functions given by (1):

$$
\sum_{n=0}^{\infty} \frac{(\lambda)_{n, k}}{n !} F_{k}(-n k, \beta ; \gamma ; x) t^{n}=(1-k t)^{\frac{-\lambda}{k}} F_{k}\left(\lambda, \beta ; \gamma ; \frac{-k x t}{1-k t}\right)
$$

where $\lambda \in C$ and $|t|<1$.

Proof. Let $T$ denote the first member of assertion (4). Using (1), we have

$$
T=\sum_{n=0}^{\infty} \frac{(\lambda)_{n, k}}{n !} \sum_{r=0}^{r<n k} \frac{(-n k)_{r, k}(\beta)_{r, k}}{(\gamma)_{r, k} r !} x^{r} t^{n}=\sum_{n=0}^{\infty} \sum_{r=0}^{r<n k} \frac{(\lambda)_{n, k}(-n k)_{r, k}(\beta)_{r, k}}{(\gamma)_{r, k} n ! r !} x^{r} t^{n}
$$

and using Lemma 2.1, we get,

$$
T=\sum_{n=0}^{\infty} \sum_{r=0}^{r<n k} \frac{(\lambda)_{n, k}(\beta)_{r, k}(-1)^{r} k^{r} n !}{(\gamma)_{r, k} n ! r !(n-r) !} x^{r} t^{n}
$$

Replacing $n$ by $n+r$ we may write that

$$
T=\sum_{n=0}^{\infty} \sum_{r=0}^{\infty} \frac{(\lambda)_{n+r, k}(\beta)_{r, k}(-1)^{r} k^{r}}{(\gamma)_{r, k} n ! r !} x^{r} t^{n+r}
$$

Using the following properties (see [4], [5])

$$
(\lambda)_{n+r, k}=(\lambda)_{r, k}(\lambda+r k)_{n, k}, \sum_{n=0}^{\infty} \frac{(\lambda)_{n, k}}{n !} t^{n}=(1-k t)^{-\frac{\lambda}{k}}
$$

we get

$$
T=(1-k t)^{-\frac{\lambda}{k}} \sum_{r=0}^{\infty} \frac{(\lambda)_{r, k}(\beta)_{r, k}}{(\gamma)_{r, k} r !}\left(\frac{-k x t}{1-k t}\right)^{r}=(1-k t)^{-\frac{\lambda}{k}} F_{k}\left(\lambda, \beta ; \gamma ; \frac{-k x t}{1-k t}\right),
$$

which completes the proof.

Theorem 2.2. We have the following generating function for the k-hypergeometric functions given by (1):

$$
\sum_{n=0}^{\infty} F_{k}(-n k, \beta ; \gamma ; x) \frac{t^{n}}{n !}=e^{t} \Phi_{k}(\beta ; \gamma ;-k x t)
$$

where $|t|<1$ and $\Phi_{k}$ is k-confluent hypergeometric function.

Proof. Let $T$ denote the first member of assertion (5). Using (1), we have

$$
T=\sum_{n=0}^{\infty} \sum_{r=0}^{r<n k} \frac{(-n k)_{r, k}(\beta)_{r, k}}{(\gamma)_{r, k} r !} \frac{x^{r} t^{n}}{n !}
$$


and using Lemma 2.1, we get,

$$
T=\sum_{n=0}^{\infty} \sum_{r=0}^{r<n k} \frac{(\beta)_{r, k}(-1)^{r} k^{r} n !}{(\gamma)_{r, k} n ! r !(n-r) !} x^{r} t^{n}
$$

Replacing $n$ by $n+r$ we may write that

$$
T=\sum_{r=0}^{\infty} \frac{(\beta)_{r, k}}{(\gamma)_{r, k} r !}(-k x t)^{r} \sum_{n=0}^{\infty} \frac{t^{n}}{n !}=e^{t} \Phi_{k}(\beta ; \gamma ;-k x t),
$$

which completes the proof.

\section{Multilinear and Multilateral Generating Functions}

In this section, we derive several families of bilinear and bilateral generating functions for the $k$-hypergeometric functions by using the similar method considered in [6]-[9].

Theorem 3.1. Corresponding to an identically non-vanishing function $\Omega_{\mu}\left(y_{1}, \ldots, y_{s}\right)$ of $s$ complex variables $y_{1}, \ldots, y_{s}(s \in N)$ and of complex order $\mu$, let

$$
\Lambda_{\mu, \psi}\left(y_{1}, \ldots, y_{s} ; \xi\right):=\sum_{l=0}^{\infty} a_{l} \Omega_{\mu+\psi l}\left(y_{1}, \ldots, y_{s}\right) \xi^{l}
$$

where $a_{l} \neq 0, \mu, \psi \in C$ and

$$
\Theta_{n, p}^{\mu, \psi}\left(x, y_{1}, \ldots, y_{s} ; \zeta\right):=\sum_{l=0}^{[n / p]} a_{l}(\lambda)_{n-p l, k} F_{k}(-(n-p l) k, \beta ; \gamma ; x) \Omega_{\mu+\psi l}\left(y_{1}, \ldots, y_{s}\right) \frac{\zeta^{l}}{(n-p l) !}
$$

Then, for $p \in N$ we have

$$
\sum_{n=0}^{\infty} \Theta_{n, p}^{\mu, \psi}\left(x, y_{1}, \ldots, y_{s} ; \frac{\eta}{t^{p}}\right) t^{n}=(1-k t)^{-\frac{\lambda}{k}} F_{k}\left(\lambda, \beta ; \gamma ; \frac{-k x t}{1-k t}\right) \Lambda_{\mu, \psi}\left(y_{1}, \ldots, y_{s} ; \eta\right)
$$

provided that each member of (6) exists.

Proof. For convenience, let $S$ denote the first member of the assertion (6). Then,

$$
S=\sum_{n=0}^{\infty} \sum_{l=0}^{[n / p]} a_{l}(\lambda)_{n-p l, k} F_{k}(-(n-p l) k, \beta ; \gamma ; x) \Omega_{\mu+\psi l}\left(y_{1}, \ldots, y_{s}\right) \frac{\eta^{l} t^{n-p l}}{(n-p l) !}
$$

Replacing $n$ by $n+p l$ we may write that

$$
\begin{aligned}
S & =\sum_{n=0}^{\infty} \sum_{l=0}^{\infty} a_{l}(\lambda)_{n, k} F_{k}(-n k, \beta ; \gamma ; x) \Omega_{\mu+\psi l}\left(y_{1}, \ldots, y_{s}\right) \frac{\eta^{l} t^{n}}{n !} \\
& =\sum_{n=0}^{\infty}(\lambda)_{n, k} F_{k}(-n k, \beta ; \gamma ; x) \frac{t^{n}}{n !} \sum_{l=0}^{\infty} a_{l} \Omega_{\mu+\psi l}\left(y_{1}, \ldots, y_{s}\right) \eta^{l} \\
& =(1-k t)^{-\frac{\lambda}{k}} F_{k}\left(\lambda, \beta ; \gamma ; \frac{-k x t}{1-k t}\right) \Lambda_{\mu, \psi}\left(y_{1}, \ldots, y_{s} ; \eta\right),
\end{aligned}
$$


which completes the proof. By using a similar idea, we also get the next result immediately.

Theorem 3.2. Corresponding to an identically non-vanishing function $\Omega_{\mu}\left(y_{1}, \ldots, y_{s}\right)$ of $s \quad$ complex variables $y_{1}, \ldots, y_{s}(s \in N)$ and of complex order $\mu$, let

$$
\Lambda_{\mu, \psi}\left(y_{1}, \ldots, y_{s} ; \xi\right):=\sum_{l=0}^{\infty} a_{l} \Omega_{\mu+\psi l}\left(y_{1}, \ldots, y_{s}\right) \xi^{l}
$$

where $a_{l} \neq 0, \mu, \psi \in C$ and

$$
\Theta_{n, p}^{\mu, \psi}\left(x, y_{1}, \ldots, y_{s} ; \zeta\right):=\sum_{l=0}^{[n / p]} a_{l} F_{k}(-(n-p l) k, \beta ; \gamma ; x) \Omega_{\mu+\psi l}\left(y_{1}, \ldots, y_{s}\right) \frac{\zeta^{l}}{(n-p l) !}
$$

Then, for $p \in N$ we have

$$
\sum_{n=0}^{\infty} \Theta_{n, p}^{\mu, \psi}\left(x, y_{1}, \ldots, y_{s} ; \frac{\eta}{t^{p}}\right) t^{n}=e^{t} \Phi_{k}(\beta ; \gamma ;-k x t) \Lambda_{\mu, \psi}\left(y_{1}, \ldots, y_{s} ; \eta\right)
$$

provided that each member of (7) exists.

\section{Special Cases}

As an application of the above theorems, when the multivariable function $\Omega_{\mu}\left(y_{1}, \ldots, y_{s}\right), l \in N_{0}$, $s \in N$ is expressed in terms of simpler functions of one and more variables, then we can give further applications of the above theorems. We first set

$$
s=r, \Omega_{\mu+\psi l}\left(y_{1}, \ldots, y_{r}\right)={ }^{(k)} E_{\left(\left\{K_{l}\right\}_{l \in N_{0} ; p, q}\right)}^{(r)}\left(\lambda+\mu+\psi l, \beta_{k+1}, \ldots, \beta_{r} ; \gamma_{1}, \ldots, \gamma_{r} ; y_{1}, \ldots, y_{r}\right)
$$

in Theorem 3.1, where the extended multivariable hypergeometric functions ${ }^{(k)} E_{\left(\left\{K_{l}\right\}_{l \in N_{0} ; p, q}\right)}^{(r)}$ generated by (see [10]):

$$
\begin{aligned}
& \sum_{n=0}^{\infty} \frac{(\lambda)_{n}{ }^{(k)} E_{\left(\left\{K_{l}\right\}_{l \in N_{0} ; p, q}\right)}^{(r)}\left(\lambda+n, \beta_{k+1}, \ldots, \beta_{r} ; \gamma_{1}, \ldots, \gamma_{r} ; x_{1}, \ldots, x_{r}\right) t^{n}}{n !} \\
& =(1-t)^{\left.-\lambda{ }^{(k)} E_{\left(\left\{K_{l}\right\}_{l \in N_{0} ; p, q}\right.}^{(r)}\right)}\left(\lambda, \beta_{k+1}, \ldots, \beta_{r} ; \gamma_{1}, \ldots, \gamma_{r} ; \frac{x_{1}}{(1-t)^{\rho}}, \ldots, \frac{x_{k}}{(1-t)^{\rho}}, \frac{x_{k+1}}{(1-t)}, \ldots, \frac{x_{r}}{(1-t)}\right) .
\end{aligned}
$$

We are thus led to the following result which provides a class of bilateral generating functions for $k$-hypergeometric functions and the extended multivariable hypergeometric functions.

Corollary 4.1. If

$$
\begin{gathered}
\Lambda_{\mu, \psi}\left(y_{1}, \ldots, y_{s} ; \xi\right):=\sum_{l=0}^{\infty} a_{l}{ }^{(k)} E_{\left(\left\{K_{l}\right\}_{l \in N_{0} ; p, q}\right)}^{(r)}\left(\lambda+\mu+\psi l, \beta_{k+1}, \ldots, \beta_{r} ; \gamma_{1}, \ldots, \gamma_{r} ; y_{1}, \ldots, y_{r}\right) \xi^{l} \\
\left(a_{l} \neq 0, \mu, \psi \in C\right)
\end{gathered}
$$


then, we have

$$
\begin{gathered}
\sum_{n=0}^{\infty} \sum_{l=0}^{[n / p]} a_{l}(\lambda)_{n-p l, k} F_{k}\left(\begin{array}{c}
-(n-p l) k, \beta \\
\gamma
\end{array} ;\right)^{(k)} E_{\left(\left\{K_{l}\right\}_{l \in N_{0} ; p, q}\right.}^{(r)}\left(\begin{array}{c}
\lambda+\mu+\psi l, \beta_{k+1}, \ldots, \beta_{r} \\
\gamma_{1}, \ldots, \gamma_{r}
\end{array} y_{1}, \ldots, y_{r}\right) \frac{\eta^{l} t^{n-p l}}{(n-p l) !} \\
=(1-k t)^{-\frac{\lambda}{k}} F_{k}\left(\lambda, \beta ; \gamma ; \frac{-k x t}{1-k t}\right) \Lambda_{\mu, \psi}\left(y_{1}, \ldots, y_{r} ; \eta\right)
\end{gathered}
$$

provided that each member of (9) exists.

Remark 4.1. Using the generating relation (8) for the extended multivariable hypergeometric functions and getting $a_{l}=\frac{(\lambda)_{l}}{l !}, \mu=0, \psi=1$ in Corollary 4.1, we find that

$$
\begin{aligned}
&\left.\sum_{n=0}^{\infty} \sum_{l=0}^{[n / p]} \frac{(\lambda)_{l}}{l !}(\lambda)_{n-p l, k} F_{k}\left(\begin{array}{c}
-(n-p l) k, \beta \\
\gamma
\end{array} ; x\right){ }^{(k)} E_{\left(\left\{K_{l}\right\}_{l \in N_{0} ; p, q}\right.}^{(r)}\right)\left(\begin{array}{c}
\lambda+l, \beta_{k+1}, \ldots, \beta_{r} \\
\gamma_{1}, \ldots, \gamma_{r}
\end{array} ; y_{1}, \ldots, y_{r}\right) \frac{\eta^{l} t^{n-p l}}{(n-p l) !} \\
&=(1-k t)^{-\frac{\lambda}{k}} F_{k}\left(\lambda, \beta ; \gamma ; \frac{-k x t}{1-k t}\right)(1-t)^{-\lambda} \\
& \quad \times{ }^{(k)} E_{\left(\left\{K_{l}\right\}_{l \in N_{0} ; p, q}\right)}^{(r)}\left(\lambda, \beta_{k+1}, \ldots, \beta_{r} ; \gamma_{1}, \ldots, \gamma_{r} ; \frac{x_{1}}{(1-t)^{\rho}}, \ldots, \frac{x_{k}}{(1-t)^{\rho}}, \frac{x_{k+1}}{(1-t)}, \ldots, \frac{x_{r}}{(1-t)}\right) .
\end{aligned}
$$

On the other hand, we set $\Omega_{\mu+\psi l}(y)=F_{k}(-(\mu+\psi l) k, \beta ; \gamma ; y)$ in Theorem 3.2, we have the bilinear generating function relations for the $k$-hypergeometric functions.

Corollary 4.2. If

$$
\begin{aligned}
\Lambda_{\mu, \psi}(y ; \xi):= & \sum_{l=0}^{\infty} a_{l} F_{k}(-(\mu+\psi l) k, \beta ; \gamma ; y) \xi^{l} \\
& \left(a_{l} \neq 0, \mu, \psi \in C\right)
\end{aligned}
$$

then, we have

$$
\begin{aligned}
& \sum_{n=0}^{\infty} \sum_{l=0}^{[n / p]} a_{l} F_{k}\left(\begin{array}{c}
-(n-p l) k, \beta \\
\gamma
\end{array} ; x\right) F_{k}\left(\begin{array}{c}
-(\mu+\psi l) k, \beta \\
\gamma
\end{array} ; y\right) \frac{\eta^{l} t^{n-p l}}{(n-p l) !} \\
& =e^{t} \Phi_{k}(\beta ; \gamma ;-k x t) \Lambda_{\mu, \psi}(y ; \eta)
\end{aligned}
$$

provided that each member of (10) exists.

Remark 4.2. Using the generating relation (5) for the k-hypergeometric functions and getting $a_{l}=\frac{1}{l !}, \mu=0, \psi=1$ in Corollary 4.2, we find that

$$
\sum_{n=0}^{\infty} \sum_{l=0}^{[n / p]} F_{k}\left(\begin{array}{c}
-(n-p l) k, \beta \\
\gamma
\end{array} ; x\right) F_{k}\left(\begin{array}{c}
-l k, \beta \\
\gamma
\end{array} ; y\right) \frac{\eta^{l} t^{n-p l}}{(n-p l) ! l !}=e^{2 t} \Phi_{k}(\beta ; \gamma ;-k x t) \Phi_{k}(\beta ; \gamma ;-k y \eta) .
$$


In the same way, we set, $\Omega_{\mu+\psi l}(y)=F_{k}(-(\mu+\psi l) k, \beta ; \gamma ; y)$ in Theorem 3.1 we have the bilinear generating function relation for the $k$-hypergeometric functions. Then again, we set, $\Omega_{\mu+\psi l}(y)=g_{\mu+\psi l}^{(s)}(y)$ in Theorem 3.2, where the Cesáro polynomials generated by [8], [9]:

$$
\sum_{n=0}^{\infty} g_{n}^{(s)}(x) t^{n}=(1-t)^{-s-1}(1-x t)^{-1}
$$

We are thus led to the following result which provides a class of bilateral generating functions for $k$-hypergeometric functions and the Cesáro polynomials.

Corollary 4.3. If

$$
\begin{gathered}
\Lambda_{\mu, \psi}(y ; \xi):=\sum_{l=0}^{\infty} a_{l} g_{\mu+\psi l}^{(s)}(y) \xi^{l} \\
\left(a_{l} \neq 0, \mu, \psi \in C\right)
\end{gathered}
$$

then, we have

$$
\sum_{n=0}^{\infty} \sum_{l=0}^{[n / p]} a_{l} F_{k}\left(\begin{array}{c}
-(n-p l) k, \beta \\
\gamma
\end{array} ; x\right) g_{\mu+\psi l}^{(s)}(y) \frac{\eta^{l} t^{n-p l}}{(n-p l) !}=e^{t} \Phi_{k}(\beta ; \gamma ;-k x t) \Lambda_{\mu, \psi}(y ; \eta)
$$

provided that each member of (12) exists.

Remark 4.3. Using the generating relation (11) for the Cesáro polynomials and getting $a_{l}=1, \mu=0, \psi=1$ in Corollary 4.3 , we find that

$$
\sum_{n=0}^{\infty} \sum_{l=0}^{[n / p]} F_{k}\left(\begin{array}{c}
-(n-p l) k, \beta \\
\gamma
\end{array} x\right) g_{l}^{(s)}(y) \frac{\eta^{l} t^{n-p l}}{(n-p l) !}=e^{t} \Phi_{k}(\beta ; \gamma ;-k x t)(1-\eta)^{-s-1}(1-y \eta)^{-1}
$$

Furthermore, for every suitable choice of the coefficients $a_{i}\left(i \in N_{0}\right)$ if the multivariable function $\Omega_{\mu+\psi l}\left(y_{1}, \ldots, y_{s}\right) \quad s \in N$ is expressed as an appropriate product of several simpler functions, the assertions of Theorems 3.1 and 3.2 can be applied in order to derive various families of multilinear and multilateral generating functions for the $k$-hypergeometric functions.

\section{References}

[1] Mubeen, S., Rahman G., Rahman A., \& Naz M. (2014). Contiguous function relations for $k$-hypergeometric functions. ISRN Mathematical Analysis, Article ID 410801, 6.

[1] Diaz, R., \& Pariguan, E. (2007). On hypergeometric functions and pochhammer k-symbol. Divulgaciones Matemáticas, 15, 179-192.

[2] Erkus, E., \& Srivastava, H. M. (2006). A unified presentation of some families of multivariable polynomials. Integral Transform. Spec. Funct., 17, 267-273.

[3] Mubeen, S., \& Rahman A. (2014). A note on $k$-Gamma Function and Pochammer $k$-symbol. Journal of Informatics and Mathematical Sciences, 6(2), 93-107.

[4] Mubeen, S. (2013). Solution of some integral equations involving confluent $k$-hypergeometric functions. Applied Mathematics, 4, 9-11. 
[5] Korkmaz-Duzgun, D., \& Erkus-Duman, E. (2018). The laguerre type $d$-orthogonal polynomials. J. Sci. Arts, 1(42), 95-106.

[6] Rainville, E. D. (1960). Special Functions. New York: The Macmillan Company.

[7] Erkus-Duman, E. (2011). Matrix extensions of polynomials in several variables. Util. Math. 85, 161-180.

[8] Ozmen, N., \& Erkus-Duman, E. (2018). Some families of generating functions for the generalized Cesaro polynomials. J. Comput.Anal.Appl. 25, 670-683.

[9] Korkmaz-Duzgun, D., \& Erkus-Duman, E. (2018). Extended multivariable hypergeometric functions. Trends in Mathematics, Research Perspectives. Springer/Birkhäuser.

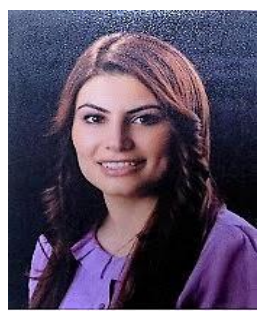

Duriye Korkmaz-Duzgun is an assistant professor of business administration at Kafkas University in Kars, Turkey. In 2017 she received the Ph.D. degree from the Department of Mathematics in Gazi University. Before she came to Kafkas University, she worked as a research assistant at Gazi University. Her research areas contain special functions, generating functions and extended hypergeometric functions.

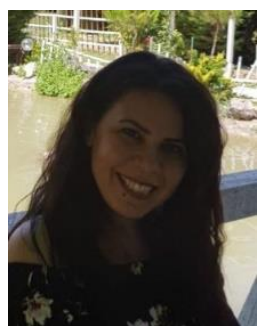

Esra Erkus-Duman is a full professor of mathematics at Gazi University in Ankara, Turkey. In 2005 she received the Ph.D. degree from the Department of Mathematics in Ankara University. Before she came to Gazi University, she worked as a research assistant at Ankara University. She became associate professor in 2010 and full professor in 2017. Her research areas contain special functions, generating functions and orthogonal polynomials. 